\title{
DOCUMENTATION OF HISTORICAL ARCHITECTURES THROUGH THE COMBINED USE OF DIGITAL SURVEYING TECHNIQUES
}

\author{
R. Valente ${ }^{1 *}$, D. Oreni ${ }^{1}$, L. Barazzetti ${ }^{1}$, F. Roncoroni ${ }^{2}$, M. Previtali ${ }^{1}$ \\ ${ }^{1}$ Politecnico di Milano, ABC Department, piazza Leonardo da Vinci, 32, 20133 Milano \\ ${ }^{2}$ Polo Territoriale di Lecco, via Previati 1/c, 23900 Lecco \\ (riccardo.valente, daniela.oreni, luigi.barazzetti, fabio.roncoroni, mattia.previtali)@polimi.it
}

KEY WORDS: Architectural surveying, laser scanning, digital photogrammetry, thermal imaging, restoration, detached frescos

\begin{abstract}
:
This paper presents the survey of the Baptistery of the Collegiata Museum (Castiglione Olona - Italy), painted by Masolino da Panicale in 1435. Surveying activity was carries out throughout the use of combined digital techniques of documentation (topographic measurements, laser scanning, digital photogrammetry, thermal imaging, digital photography). Different data were collected inside and outside the Baptistery following a multi-resolution reconstruction that ranges from the whole Baptistery up to small details. Laser scans were also used to obtain a digital representation of inner volumes of the structure. Registered scans were uploaded into a modelling software, in order to produce a 3D model based on scan data, increasing the correspondence with the existent building; this model can be used to better understand the volume arrangement, also for educational and dissemination purposes. A particular attention was devoted to documentation of graffiti scratched onto the frescos, since their presence is a significative evidence of the history of the building. A digital-based workflow was set out in order to assure high quality outputs in respect of the conservation needs.

This multi-resolution approach proved to be a suitable solution when dealing with features, such as ancient architectures, with a high number of complex details to be correctly recorded and joint together as starting point and support of historical researches. Results and scope are here presented.
\end{abstract}

\section{INTRODUCTION}

The last decade was distinguished by a series of digital revolutions that involved methods, techniques and instruments for both conservation and surveying. Both disciplines took great advantages by these processes of digitalization, resulting in new opportunities of research and data analysis. Moreover, many surveying activities are specifically aimed at conservation or restoration purposes. Here the results of the combined use of digital techniques are briefly presented, with a special attention to aspects that involve conservation aspects. Digital surveying techniques revolutionized the surveying practice in the last decades, replacing in many cases traditional methods. Two of the most used digital technologies in architectural surveying are digitap photogrammetry and laser scaning. Terrestrial and aerial digital photogrammetry has been extensively used in the last years to document Cultural Heritage in the wider sens, from built structures, to archaeological sites (D'Aprile and Piscitelli, 2019). Laser scanning, based on different laser impulse devices, can record millions of measured points in a short span of time, obtaining 3D point clouds which can be successfully used to extract plan and section drawings; laser scanning is particularly useful when dealing with historical structures due to their intrinsic complexity which is sometimes hard to record in every existent detail (Barazzetti et al., 2017). Another useful technology which has been applying to Cultural Heritage field for years to analyse and assess ancient buildings is thermal imaging. Thermal imaging is based on the detection of long-infrared wavelength (Vollmer and Möllman, 2017, Lagüela et al., 2016). Thermal images are particularly useful to detect a number of features that are not immediately perceivable by naked eye. In historical buildings this means for

\footnotetext{
* Corresponding author
}

instance: moisture absorption, which could cause severe damages to walls (Ludwig, Rosina and Sansonetti, 2018), or the individuation of hidden elements such as the masonry patterns, which is often covered by plaster (Spodek and Rosina, 2009).

\section{CASE STUDY}

The Baptistery of the Collegiata in Castiglione Olona (Italy) is one of the most relevant architectural and artistic sites of Lombardy region. Its unicity is given by the fact that most of the 15th century frescos were painted by Masolino da Panicale and other Tuscan painters such as Lorenzo di Pietro and Paolo Schiavo: due to this reason, the entire complex, founded by Cardinal Branda Castiglioni upon a natural hill, is also known as the "Island of Tuscany in Lombardy".

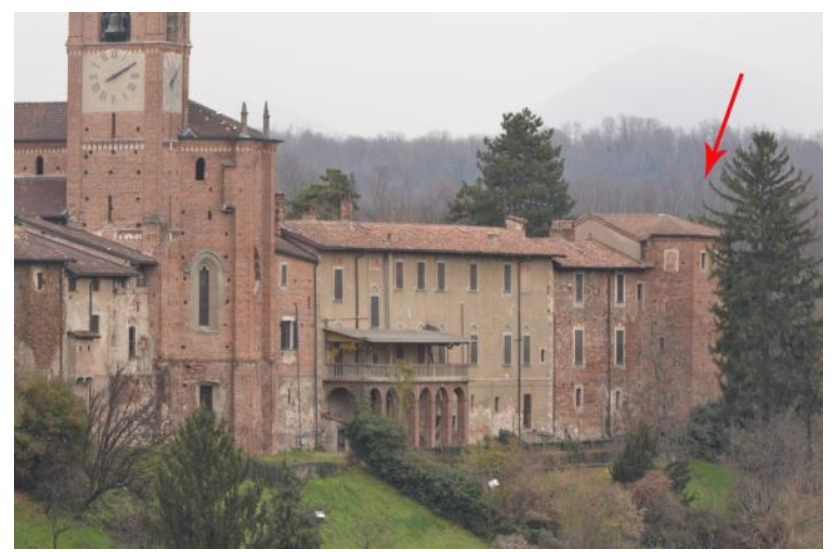

Figure 1: The ancient tower where the Baptistery is located 
The Baptistery was realized into a former castle tower, adapting the existing spaces (fig. 1). The exterior aspect is mostly covered by other close structures, except for the back area corresponding to the external front of the tower towards the valley floor. The inner part is composed of only one inner room, separated into two connected spaces of different size: the first one, has a square plan covered by a cross vault, the second one, smaller, has a rectangular plan and is covered by a pointed barrel vault; the two spaces are connected through a small triumphal arch (fig. 2).

All the interior room is decorated with frescos, painted by Masolino by the year 1435, with stories of the life of St. John the Baptist on the walls and the Four Evangelists (Dallaj, 1986, Bertelli, 1997, Joannides, 1988). There is also an inferior floor, actually not accessible, that follows the same plan of the superior room; no decorations are visible on this floor, and probably were never realized. The entire cycle suffered the negative action of moisture and other biological agents (Gallone, 1997). Moisture damages are particularly localized on the northern walls, due to climatic conditions and the presence of a close wooded area. Several restorations were carried out over the years, in order to minimize these effects. In 1876 the external walls were restored and partially rebuilt, while an underpinning of the tower foundations was realized in 1917. Other interventions were carried out in 1935 during the five hundredth anniversary of the cycle. Despite the efforts, the frescoed decoration of northern part was almost completely lost, due to the aforementioned issues.

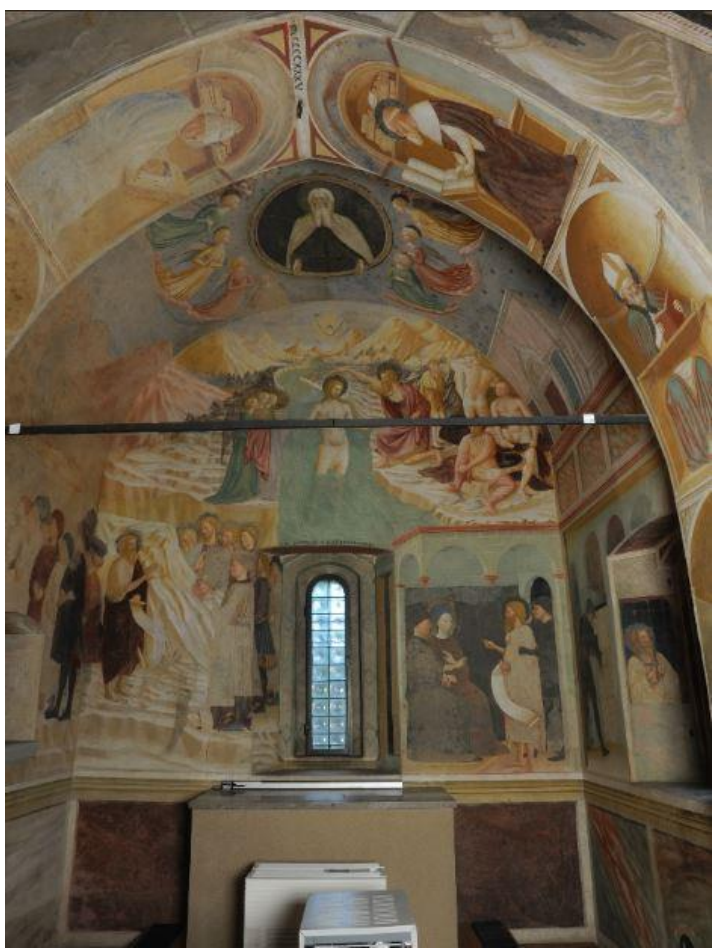

Figure 2: the interior of the Baptistery decorated with Masolino's frescos

Between 1964 and 1971 were detached for preservation purposes some frescos localized on the external front, on the north and west inner wall and on the two vaults. The last restoration campaign was carried out between 1995 and 1999 (Brambilla Barcilon, 1997); an automatic system of moisture and air control is currently active inside of the Baptistery. The previous restorations preserved also a large number of ancient graffiti, which are an important evidence and part of the history of the monument (Valente, 2013).

Despite the relevance of the monument, no up-to-date surveys were available for the Baptistery. One of the aims of the new surveying campaign was not just producing new outputs, such as plans and sections, but also to try to collect as much data as possible in terms of quality and detail, with a particular attention to preservation and restoration aspects.

The focus was set only on the inner part of the structure, since the outer parts were partially covered by other structures and the surrounding space was not easily accessible due to terrain obstacles (steep hillsides and woods). There were no critical points about the size or complexity of the structures, but on the amount of important details to be correctly recorded. Among these ones, two aspects revealed to be particularly important for the history of the structure: the traces of previous restoration campaigns and the relevant presence of ancient graffiti. Besides the geometric surveying, these two peculiarities were especially addressed during the measurement campaign.

\section{FIELD SURVEYING AND DATA PROCESSING}

\subsection{Field surveying}

Most of the geometric surveying relied on the use of laser scanning. It's been a consolidated technique for the architectural surveying for almost a decade; improvements in technology allowed for more powerful and portable instruments, able to record some millions of points for every scan. A series of scans with colour information were taken inside the Baptistery with a Faro Focus 3D. Registration was carried out with targets obtaining a precision of about $\pm 2-3 \mathrm{~mm}$ and 220 million points inside the structure $(8 \times 4 \times 6 \mathrm{~m}$ roughly). Both checkboard and sphere targets were used; the instrument was able to capture also some architectural details, such as window profiles, which are usually more likely to produce a nodata area. Registration was carried out using Scene software, which allows automatically detection of targets (fig. 3).

The use on the field of a total station Leica 1203 allowed to correctly measure the laser target, assuring al rigorous local reference system, and other control points on the frescos.

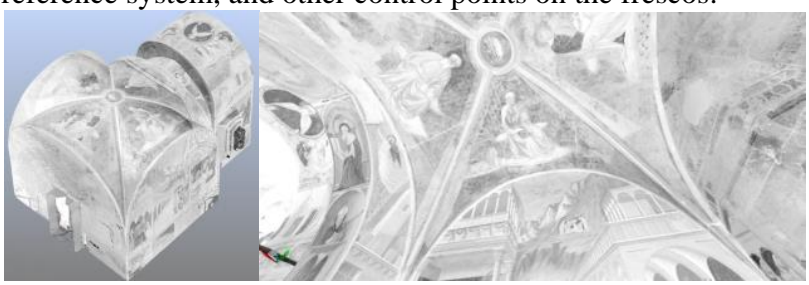

Figura 3: Laser scans of the interior space of Baptistery (grey scale visualization)

In order to assure a complete photographic documentation of inner frescos, several images of every wall were taken. Both rectified images and orthophotos of the most interesting portions were planned. Two methods were adopted in this task: a standard photogrammetric image block automatically oriented (strips of normal images coupled with convergent images to strengthen network geometry) and the gnomonic projection method where a high resolution image is created by stitching single images taken with a rotating camera. A double system of lighting was used: diffuse, to better capture colour information, and raking, to enhance marks and other surface discontinuities. Additional detailed images of single ancient graffiti were also 
acquired, to be used as a base for digital drawings; these sets of images were principally acquired with raking lighting, so as to enhance their visibility and readability.

In this case a series of premeasured frames were used to obtain a more accurate georeferencing (fig. 4).

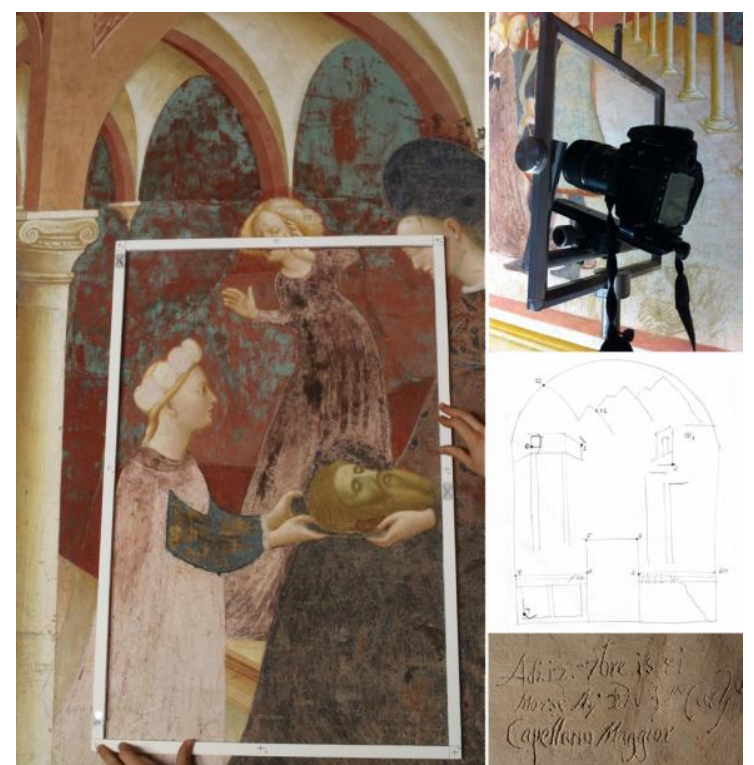

Figure 4: Photographic documentation of graffiti and manual sketches of measurements

A test was carried out also to verify the conditions of detached frescos. After detachment, the paint layers were arranged on large canvas and then positioned on frameworks to be placed back in the original position on the inner walls of Baptistery. One detached fresco, a figure of a Sybil, was mounted on a mobile framework, so that is still possible to move it and to observe the underlying sinopia (preparatory drawing). Since the other frescos were permanently fixed to the wall, a thermographic inspection of the cross vault was carried out using a FLIR Tau portable thermal camera. Thermal imaging can easily reveal hidden features in standing walls and structures and was already successfully tested on monitoring of frescos Rizzi et al., 2007, Cadelano et al., 2015).

\subsection{Data processing}

Data collected on the field were successively processed. Laser scans were used to produce $2 \mathrm{D}$ and $3 \mathrm{D}$ outputs. Registered cloud points were uploaded into a CAD software where an horizontal slice corresponding to the Baptistery plan was created and retraced. The same scans were also uploaded into Rhinoceros, a modelling software, after their conversion into an interoperable file format.
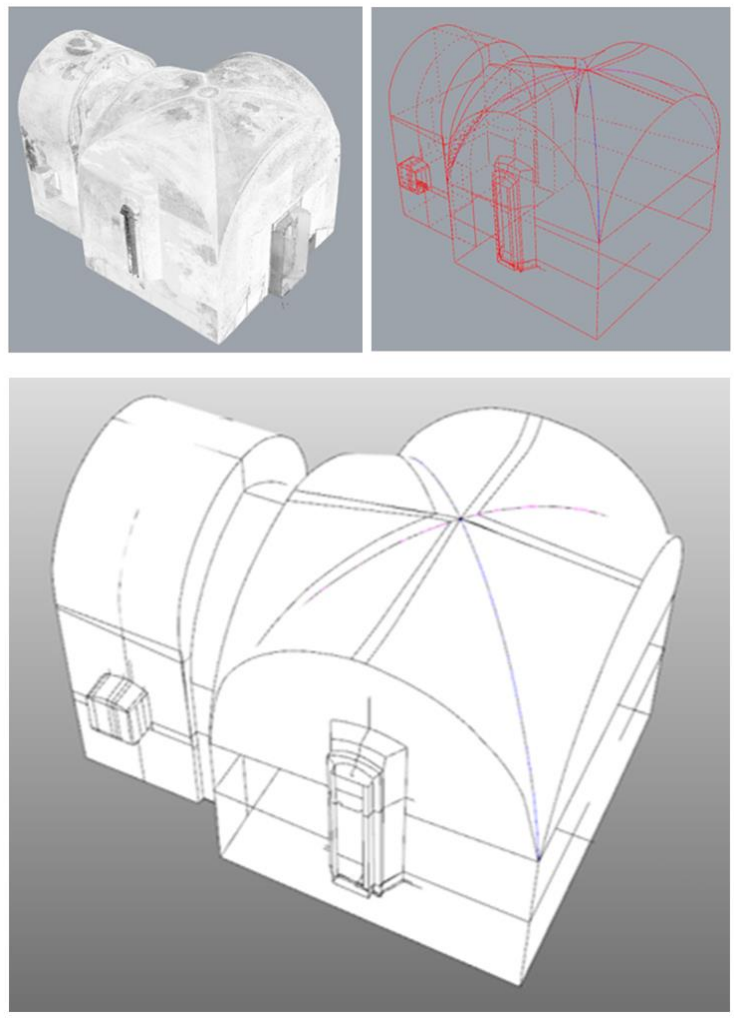

Figure 5: Laser scans (above), generative lines of the structure (middle), 3D model composed by digital surfaces

A specific plug-in (Point Cloud) for point clouds produced by laser scanners was used in order to better manage them. A series of different section lines were traced with polylines alongside the major generative lines of the structure. Thanks to these polylines, the respondent wall surfaces were digitally generated, with a high correspondence with laser scans and real object (fig. 5). For what concerns the photographic documentation, a series of rectified images of single walls were created. Since most of the inner wall are sub-planar surface, operations of rectification aimed to minimize distortions were successful. The different rectified images were successively assembled together in three-dimensions, using topographic measurements as reference and recreating the spatial disposition of walls. Two large portions of the southern wall, where there are most of the ancient graffiti, were also selected for a more detailed documentation. In this case, four different photogrammetric projects were set, two for each wall with different lighting conditions. The image blocks were processed with Agisoft PhotoScan Pro (fig. 6). 


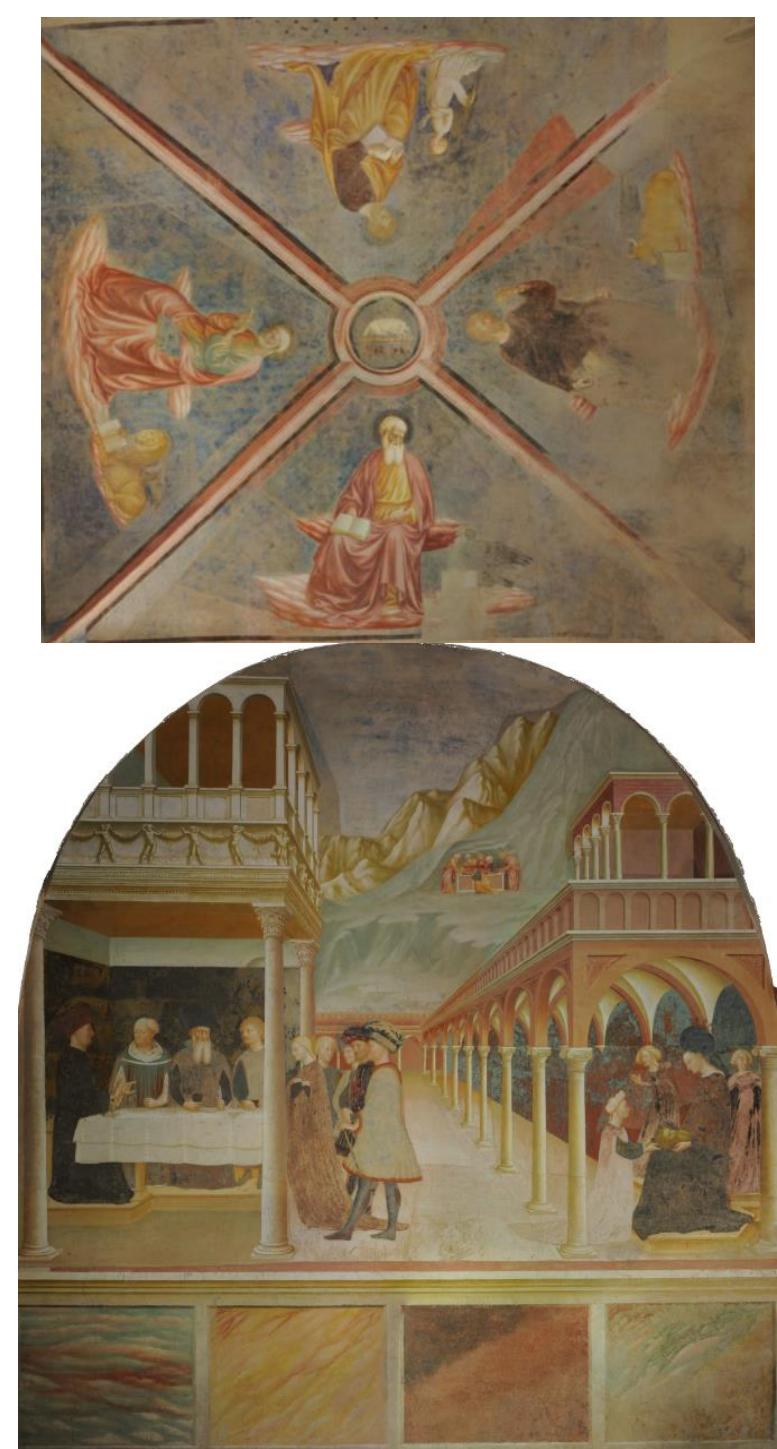

Figure 6: Orthophotos of the main vault (left) and of southern wall (right)

\section{RESULTS AND DISCUSSION}

The several 2D and 3D outputs obtained from laser scans helped to better understand the architecture and to update the existent documentation. Their creation was simplified by the use of cloud points, which allows for saving more time on the field and have a greater amount of data compared to only traditional total station surveying.

Detailed orthorectified of wall portions with ancient graffiti were used to obtain digital drawings, as already presented in a previous work (Valente and Oreni, 2017). This digital copies made possible to display both the morphology of graffiti as well as their interpretation, not missing also secondary marks or surface damages.

Some important results were obtained analysing the thermal images of the main vault of the Baptistery. Today, only few visible clues suggest that the entire decoration was detached and then put in place again: for instance, a small rim is detectable along the external edge of vault frescos, where there is the connection with vertical walls. Thermal images revealed more than what can be currently see: near infrared sensor clearly shows geometrical lines which do not belong to any decorative or architectural element. This pattern is a hidden structure underneath the frescos and must be the rigid frame where the canvas with detached frescos are fixed. The structure appears as a regular grid of squared elements, possibly made out of metal or wood (fig. 7).

The regular pattern is interrupted only along the arrises of the vault. In this area, larger profiles are visible, probably because here is where the different vault surfaces joint and where the frame is probably fixed to the vault itself. Similar larger profiles are visible on the external edges. A decorated band is present on the profiles of both arrises and external edges, but the features visible on the thermal images should not belong to frescos, because no other decorations, such as the Evangelists' figures, are detected.
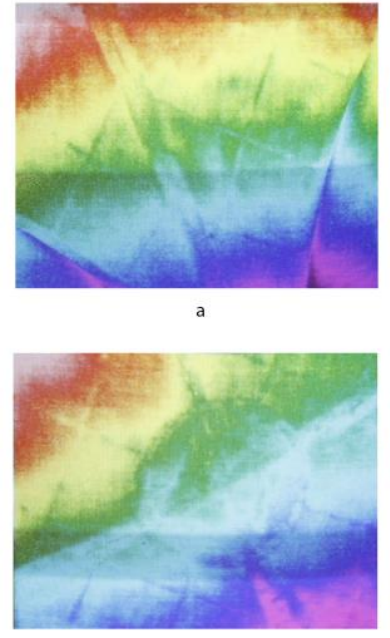

c

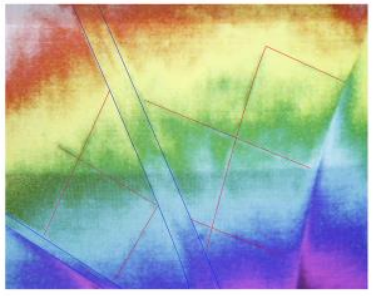

b

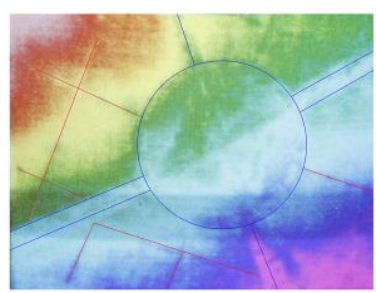

d
Figure 7: Enhancement of hidden lattice as support for detached frescos

It is likely that the framework elements have similar sizes to decorations, maybe a method to better hide their presence. Another interesting part is the central part of the vault, where there is a circular decoration with an Agnus Dei depicted. The circular edge is still visible also on the thermal images, and this could suggest the presence of another hidden structure, where the vault is almost flat.

\section{CONCLUSIONS}

The surveying and tests carried out in the Baptistery of Castiglione Olona proved the efficiency of combining different digital techniques to record different aspects of historical architectures. Laser scanning with the support of topographic measurements allows for obtaining reliable $2 \mathrm{D}$ and $3 \mathrm{D}$ representations of the geometry of the structure. Digital photogrammetry and rectified images were used to get high quality images that could also used as metric reference. Varying the lighting conditions of photogrammetric blocks was also useful to detect ancient graffiti and other interesting aspects such as the portions of fresco painted in different days. Their digital contactless documentation was extremely useful, both for recording and conservation as well as for study purposes. Thermal images also proved to be very useful to detect the traces of previous restorations, in this particular case of detachment of frescos. This method could be used also to monitor the structure that physically supports the canvas, since any possible failure due to age or other factors could be hard to be individuated by simple visual inspection. 


\section{REFERENCES}

Agisoft PhotoScan, http://www.agisoft.com/.

Barazzetti, L., Mezzino, D., Santana Quintero, M., 2017. Digital Workflow for the Conservation of Bahrain Built Heritage: The Sheik Isa Bin Ali House. ISPRS - International Archives of the Photogrammetry, Remote Sensing and Spatial Information Sciences. XLII-2/W5, 65-70. doi.org/10.5194/isprsarchives-XLII-2-W5-65-2017.

Bertelli, C., 1997. Masolino, gli affreschi del Battistero e della Collegiata a Castiglione Olona. Milano.

Brambilla Barcilon, P., 1997. Studi preliminari sugli affreschi di Masolino da Panicale a Castiglione Olona. Conservazione $e$ valorizzazione degli affreschi nella Provincia di Varese, ed.by P. C. Marani. Varese, 35-41.

Cadelano, G., Bison, P., Bortolin, A., Ferrarini, G., Peron, F., Girotto, M., Volinia, M., 2015. Monitoring of historical frescos by timed infrared imaging analysis. Opto-electronics Review, 23(1), 100-106.

Dallaj, A., 1986. Masolino da Panicale. Le storie di Maria e del Battista a Castiglione Olona, destinazione e schemi compositivi. Milano.

D'Aprile, M. and Piscitelli, M., 2019. Survey, Stratigraphy of the Elevations, 3D Modelling for the Knowledge and Conservation of Archaeological Parks: the Castle of Avella, Int. Arch. Photogramm. Remote Sens. Spatial Inf. Sci., XLII-2/W9, 289-296, https://doi.org/10.5194/isprs-archives-XLII-2W9-289-2019.

Gallone, A. V., 1997. Analisi di campioni di colore prelevati dagli affreschi di Masolino da Panicale a Castiglione Olona. Conservazione e valorizzazione degli affreschi nella Provincia di Varese, ed.by P. C. Marani. Varese, 43-47.

Joannides, P., 1988. Masolino a Castiglione Olona: il Battistero e la collegiata. Arte in Lombardia tra Gotico e Rinascimento. Milano.

Lagüela, S., Díaz Vilariño, L., Roca, D., 2016. Infrared Thermography: Fundamentals and Applications. Nondestructive Techniques for the Evaluation of Structures and Infrastructres, ed. By B. Riveiro - M. Solla. 113-138. doi.org/10.1201/b19024-8.

Ludwig, N., Rosina, E., Sansonetti, A., 2018. Evaluation and monitoring of water diffusion into stone porous materials by means of innovative IR thermography techniques.

Measurements, 118, 348-353.

Previtali, M., Barazzetti, L., Redaelli, V., Scaioni, M., Rosina E., 2013. Rigorous procedure for mapping thermal infrared images on three-dimensional models of building façades. Journal of Applied Remote Sensing, 7.1, 1-20.
Rizzi, A., Voltolini, F., Girardi, S., Gonzo, L., Remondino, F., 2007. Digital Preservation, Documentation and Analysis of Paintings, Monuments and Large Cultural Heritage with Infrared Technology, Digital Cameras and Range Sensors. XXI International CIPA Symposium, 01-06 October 2007, Athens, Greece.

Spodek, J., Rosina, E., 2009 Application of Infrared Thermography to Historic Building Investigation, Journal of Architectural Conservation, 15:1, 65-81. doi.org/10.1080/13556207.2009.1078504.

Valente, R., 2013. I graffiti del Battistero di Castiglione Olona. Aevum, 87.3, 807-874.

Valente, R. and Oreni, D., 2017. Survey and Drawings for the study and enhancement of ancient wall graffiti: the case of the Baptistery of Castiglione Olona. DISEGNARECON, 10.19, 7.17.10 .

Vollmer, M., Möllmann, K.-P., 2017. Infrared Thermal Imaging: Fundamentals, Research and Applications. 